\title{
Epidemiological Pattern of Non-Fermenting Metallo Beta Lactamase Producing Bacterial Pathogens Isolated From Clinical Specimens in a Tertiary Care Hospital in Kakinada
}

\section{Usha Rani P and Vijayalakshmi P*}

Department of Microbiology, Gitam Institute of Medical Sciences and Research, Gitam University, India

*Corresponding author: Payala Vijayalakshmi, Department of Microbiology, Gitam Institute of Medical Sciences and Research, Gitam University, India

Received: December 01, 2016; Accepted: February 20, 2017; Published: February 22, 2017

\begin{abstract}
Background: Non-fermentative Gram negative bacilli are known causative pathogens responsible for hospital associated infection and for high morbidity and mortality rate across all genders and ages.

Objectives: This study examined the prevalence of Non-Fermentative Gram Negative Bacilli Metallo Beta-Lactamase Isolates (NFGNB-MBL) among carbapenem-resistant strains from clinical specimens and the epidemiological pattern (Age, Sex and Source of infection).

Methodology: A total of 250 clinical specimens from diverse infections were collected and analyzed, using standard microbiological methods. For MBL detection, two previously described methods were employed i.e. ImipenemEDTA combined disc test and Imipenem-EDTA double disc synergy test (DDST).

Results: Sixty (24\%) NFGNB isolates were identified, highest number from hospital associated infections (93.33\%). Pseudomonas aeruginosa accounted for $n=40(66.67 \%)$ followed by Acinetobacter baumannii $n=10 \quad(16.67 \%)$, Alcaligenes faecalis $n=6(10 \%)$ and Stenotrophomonas maltophilia $n=4(6.66 \%)$ respectively. The isolates showed high sensitivity to imipenem (91.67\%) and the incidence of MBL producing non-fermenters among the imipenem resistant organisms was found to be $100 \%$. Conclusion: The prevalence of MBL producing isolates and association with hospital infection is of clinical concern and the therapeutic option in treatment and management of patient.
\end{abstract}

Keywords: Non-fermenters; Gram negative bacilli; Antibiotic sensitivity; Imipenem; MBL production

\section{Introduction}

Non-fermenting Gram negative bacilli are a group of aerobic, non-sporing organisms that either do not use carbohydrates as a source of energy or degrade them through metabolic pathways other than fermentation [1]. These organisms are abundant in nature like soil, water, plants, decaying vegetation, food stuffs and normal flora of humans. Although NFGNB are considered commensals or contaminants, their pathogenic potential has been well established by their frequent isolation from clinical samples and their association with clinical disease. The out breaks of nosocomial infections, emerging antimicrobial resistance and epidemiology complexity have made NFGNB the remarkable organisms. NFGNB are innately resistant to many antibiotics and are known to produce extended spectrum beta-lactamase and metallo-beta-lactamase. They account for around $15 \%$ of all bacterial isolates from clinical samples. Most of the Non-fermenting Gram negative bacilli have emerged as important nosocomial pathogens in hospitalized patients of severe burns, urinary tract infections, wound infections, septicaemia, pneumonia, osteomyelitis, peritonitis, meningitis, cirrhosis of liver etc. Some can cause serious infections in immuno-compromised hosts [2]. The most important members are Pseudomonas followed by Acinetobacter, Stenotrophomonas, Moraxella, Alcaligenes, Flavobacterium, Achrobacterium etc. All these may be acquired nosocomially, so that rapid identification and epidemiological characterization are important in tracking hospital outbreaks. The rising incidence of Pseudomonas infection in hospital and its ubiquitous association in hospital environmental soil and water had led to an increased interest in identifying the strain by various bacteriological and biochemical properties by different research workers. Non-fermenting Gram negative bacilli were isolated from blood cultures of patients admitted to high risk units like oncology, nephrology, burns, NICU and cardiology [3]. With particular references to ICU and major superficial and systematic infections, Pseudomonas aeruginosa is the most common isolate followed by Acinetobacter and Alcaligenes. Bacteraemia due to Pseudomonas and Acinetobacter has become an important cause of morbidity and mortality in hospital association infections. Prolonged hospital stay, more invasive procedures for diagnosis and therapy, indiscriminate use of broad spectrum antibiotics and underlying host factors also contribute to morbidity and mortality.
J Bacteriol Mycol - Volume 4 Issue 1 - 2017

Vijayalakshmi et al. (C) All rights are reserved
Citation: Usha Rani P and Vijayalakshmi P. Epidemiological Pattern of Non-Fermenting Metallo Beta Lactamase Producing Bacterial Pathogens Isolated From Clinical Specimens in a Tertiary Care Hospital in Kakinada. J Bacteriol Mycol. 2017; 4(1): 1043. 
Table 1: Distribution of cases from different types of infections according to the age.

\begin{tabular}{|c|c|c|c|c|}
\hline S.NO & Age & Number of cases & Percentage & \\
\hline 1 & & $1-9$ & 15 & 6 \\
\hline 2 & & $10-19$ & 24 & 9.6 \\
\hline 3 & $20-29$ & 58 & 23.2 \\
\hline 4 & $30-39$ & 52 & 20.8 \\
\hline 5 & $40-49$ & 27 & 10.8 \\
\hline 6 & & $50-59$ & 39 & 15.6 \\
\hline 7 & & $60-60$ & 25 & 10 \\
\hline 8 & & $70-79$ & 10 & 4 \\
\hline & Total & 250 & & \\
\hline
\end{tabular}

In the present study an attempt has been made for isolation and identification of various non-fermenting gram negative bacilli and to study the various biochemical characters of isolated non-fermenting organisms from various clinical materials. As some of the nonfermenting organisms show multidrug resistance, the nosocomial outbreaks of carbapenem resistant non-fermenters is due to metallobeta-lactamase enzyme production. So this enzyme is responsible for multidrug resistance, and they possess high hydrolytic activity that leads to degradation of higher generation cephalosporins. Plasmid mediated MBL genes spread rapidly to other species of gram negative bacilli, therefore rapid detection of metallo-beta-lactamase production is necessary to modify therapy and to initiate effective infection control to prevent their dissemination [4]. So identification of metallo- beta-lactamase producing non-fermenters was also included in this study.

\section{Materials and Methods}

Different clinical samples collected from both IP and OP patients in Tertiary care hospital, Kakinada for a period of one year, constituted the material for this study. A total of 60 non-fermenters isolated from different clinical specimens including Pus, Urine, Endotracheal aspirates, Sputum, Blood, Catheter tips, Ascitic fluid, Pleural fluid and CSF from 250 patients. The cases included individuals of both sexes and all age groups. Gram staining was performed initially to study the morphological characteristics of the clinical isolates [5].

\section{Culture and biochemical reactions}

All the clinical specimens were initially processed to separate the non-fermenters from the other Gram negative bacilli.

Pus, respiratory secretions and other specimens were inoculated onto 5\% blood agar, Mac-conkey agar and Nutrient agar and urine samples were inoculated into CLED agar and incubated aerobically at $37^{\circ} \mathrm{C}$ for $24 \mathrm{~h}$ and then examined for growth. The wound swabs are inoculated into nutrient broth and incubated for $3-4 \mathrm{~h}$ at $37^{\circ} \mathrm{C}$ and then after noting the turbidity due to growth sub cultured onto MacConkey, 5\% blood agar and incubated for $12-18 \mathrm{~h}$ at a temperature of $37^{\circ} \mathrm{C}$. In case of Acinetobacter growth on MacConkey medium showed a faint pink tint, while no pigmentation was seen on blood agar. After observing the motility of organisms, Gram stain was done and confirmed that the organisms are gram negative.

Non fermenting organisms are provisionally identified by colonial morphology and pigment production and these were subjected to various biochemical and biological tests for confirmation [5]. The tests include carbohydrate fermentation tests with glucose, lactose, sucrose, xylose, mannitol and maltose. The organisms were also tested for Indole production, Methyl red test, Voges-proskauer test, Citrate utilization, Urease test, Oxidase test, Catalase test, Nitrate reduction test, Triple sugar iron agar test, Hughs Leifsons test (O/F test), Gelatin hydrolysis, Amino acid decarboxylation test, Growth on nutrient agar containing $6 \% \mathrm{NaCl}$, growth at $42^{\circ} \mathrm{C}$ etc. In addition to the biochemical reactions, strains of Pseudomonas aeruginosa were further tested for their ability to grow on cetrimide (0.3\%) agar. Pseudomonas aeruginosa was identified by its colony characters, pigment production and its characteristic odour, growth on cetrimide agar with $6 \% \mathrm{NaCl}$.

\section{Antibiotic sensitivity testing of isolated Non-fermenters}

Overnight broth culture having semi-confluent growth of the isolated bacterial strain was used as inoculums. Muller-Hinton agar plates were used and subjected to antibiotic susceptibility test using Disc-diffusion method of Kirby-Bauer, using the concentration of antibiotics per discs, recommended by the WHO experts committee on biological standardization. The antibiotics used were Amikacin $(30 \mu \mathrm{g})$, Gentamicin $(10 \mu \mathrm{g})$, Cefoperazone $(75 \mu \mathrm{g})$, Ceftazidime $(30 \mu \mathrm{g})$, Ceftriaxone $(30 \mu \mathrm{g})$, Ciprofloxacin $(5 \mu \mathrm{g})$, Imipenem $(10 \mu \mathrm{g})$, Piperacillin/Tazobactum (100/10 $\mu \mathrm{g})$, and Polymyxin B (300U). Results were interpreted in Zone of inhibition and comparing with

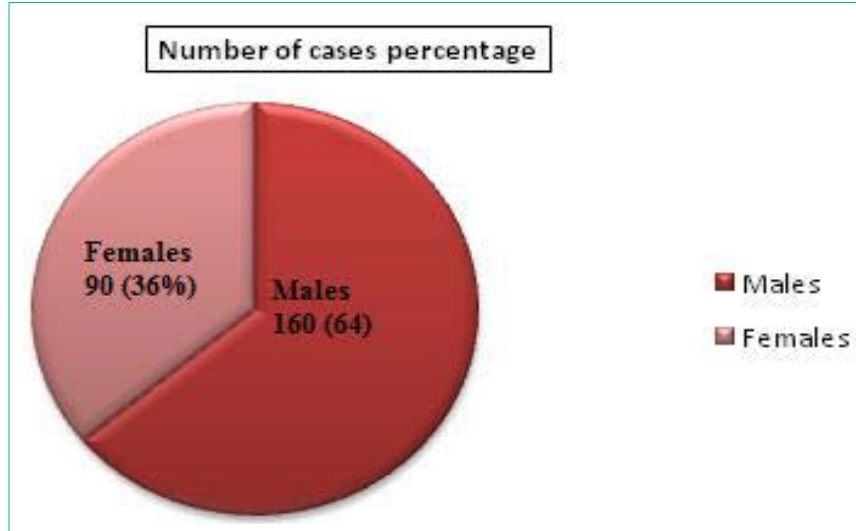

Figure 1: Distribution of cases from various types of infections according to sex.

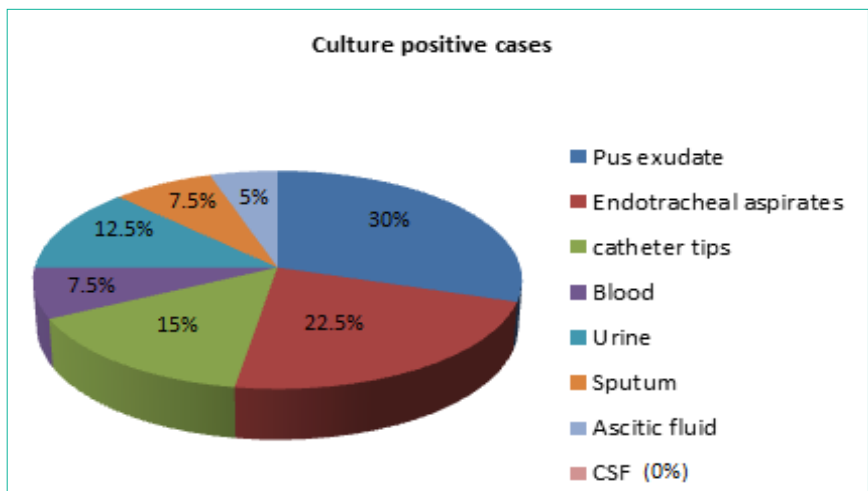

Figure 2: Distribution of isolates of Pseudomonas aeruginosa from various clinical samples. 
Table 2: The differentiating features utilized for the isolates.

\begin{tabular}{|c|c|c|c|c|c|c|}
\hline S.No & Test & $P$. aeruginosa & A. baumannii & A. faecalis & S. maltophilia & \\
\hline 1 & & Gram stain & Gram negative rod & Gram negative coccobacilli & Gram negative rod & Gram negative rod \\
\hline 2 & & Motility & Motile & Non-motile & Motile & Motile \\
\hline 3 & & Catalase & Positive & Positive & Positive & Positive \\
\hline 4 & & Oxidase & Positive & Negative & Positive & Negative \\
\hline 5 & & Growth on MacConkey agar & Non-lactose fermenter (NLF) & Faint pink tint & NLF & NLF \\
\hline 6 & & Haemolysis on $5 \%$ blood agar & Positive & Negative & Variable & Positive \\
\hline 7 & & Pigment production on nutrient agar & Positive & Negative & Negative & Positive \\
\hline 8 & & Growth at $37^{\circ} \mathrm{C}$ & Positive & Positive & Positive & Positive \\
\hline 9 & & Growth at $42^{\circ} \mathrm{C}$ & Positive & Positive & Positive & Negative \\
\hline 10 & & Indole test & Negative & Negative & Negative & Negative \\
\hline 12 & & Voges-proskauer test & Negative & Negative & Negative & Negative \\
\hline 13 & & Citrate utilization test & Positive & Negative & Positive & Positive \\
\hline 14 & & Urea hydrolysis & Variable & Variable & Negative & Negative \\
\hline 15 & & Nitrate reduction test & Positive & Negative & Negative & Variable \\
\hline 16 & & NO3to gas & Positive & Negative & Negative & Negative \\
\hline 17 & & O/F glucose & Positive & Positive & Negative & Positive \\
\hline 18 & & O/F lactose & Negative & Positive & Negative & Variable \\
\hline 19 & & O/F Maltose & Positive & Negative & Negative & Positive \\
\hline 20 & & Arginine decarboxylation & Positive & Positive & variable & Negative \\
\hline 21 & & Lysine decarboxylation & Negative & Negative & variable & Positive \\
\hline 22 & & Gelatin hydrolysis & Positive & Variable & Negative & Positive \\
\hline
\end{tabular}

the standard zone size given by Kirby-Bauer chart [5].

\section{Detection of metallo beta lactamase (MBL) production}

Screening for MBL production was done in Multidrug resistant isolates of the non-fermenters. MDR isolate was defined as resistant to 2 or more drugs or drug classes of therapeutic relevance. Among these some carbapenem resistant isolates MBL producers [6]. The following methods were used for the MBL detection in the present study which includes Imipenem-EDTA combined disc test and Imipenem-EDTA Double Disc Synergy Test (DDST). ImipenemEDTA combined disc test, the test organisms were inoculated onto

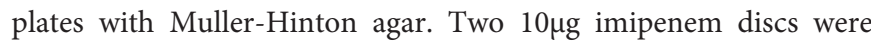
placed on the plate, an appropriate amount of $10 \mu$ l of EDTA solution were added to one of them to obtain the desired concentration $(750 \mu \mathrm{g})$. The inhibition zones of the imipenem-imipenem and EDTA discs were compared after $16-18 \mathrm{~h}$ incubation at $37^{\circ} \mathrm{C}$. In the combined disc test, if the increase in inhibition zone with the imipenem and EDTA was $>7 \mathrm{~mm}$ than the imipenem disc alone, it was considered as MBL positive [8]. Discs were prepared by adding EDTA solution to $10 \mu \mathrm{g}$ imipenem discs to obtain a concentration of $750 \mu \mathrm{g}$. The discs were dried immediately in an incubator and stored at $4^{\circ} \mathrm{C}$ or at $-20^{\circ} \mathrm{C}$ in an airtight vial without desiccant. Test strains were adjusted to the Mc Farland 0.5 standard and were inoculated to $\mathrm{MH}$ agar. A $10 \mu \mathrm{g}$ imipenem discs and an imipenem + EDTA 750 $\mu$ g were placed on $\mathrm{MH}$ agar. Another disc containing only $750 \mu$ g EDTA was also placed as a control. After overnight incubation, the established zone diameter difference of $>7 \mathrm{~mm}$ between imipenem discs and imipenem + EDTA was interpreted as EDTA synergy positive. These were compared with ATCC 27853 strains of Pseudomonas aeruginosa as a negative control [6,7].

The Imipenem-EDTA double disc synergy test was performed as described by Lee et al. Test organisms were inoculated onto plates with $\mathrm{MH}$ agar as recommended by the CLSI. An imipenem (10 $\mu \mathrm{g})$ disc was placed $20 \mathrm{~mm}$ centre to centre from a blank disc containing $10 \mu \mathrm{l}$ of $0.5 \mathrm{M}$ EDTA $(750 \mu \mathrm{g})$. Enhancement of the zone of the inhibition in the area between imipenem and the EDTA disc in comparison with the zone of inhibition on the far side of the drug was interpreted as a positive result [8].

\section{Results}

Of the 250 clinical specimens analyzed, 60 (24\%) were identified as Non-fermentative Gram negative bacilli. These 60 were used for further processing. Various age groups ranging from 1 and 79 years of both sexes were included in the present study. Out of 250 patients, 160 were males and 90 were females. The analysis of these cases in different age group is represented in (Table 1). From the table it was noticed that, different types of infections in the age group 20-29, that is $58(23.2 \%)$ and a lower number of cases in the age group 70-79, which is 10 (4\%). The rate of infection was more in males 160 (64\%) while comparing with females 90 (36\%) who attended the hospital from various infections (Figure 1).

Depending on the source, the infections were categorized into Hospital acquired and Community acquired. The number of culture 


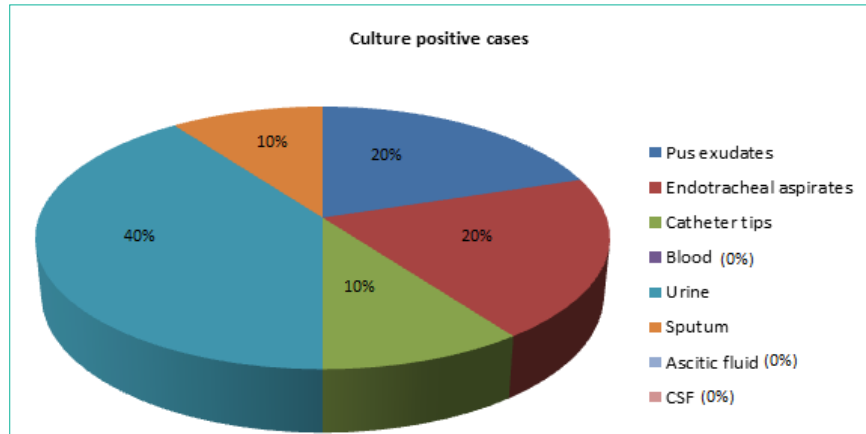

Figure 3: Distribution of isolates of Acinetobacter baumannii from various clinical samples.

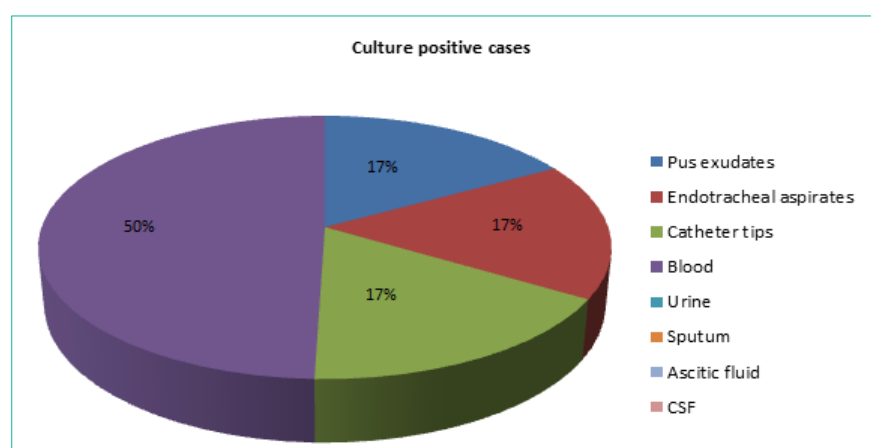

Figure 4: Distribution of isolates of Alcaligenes faecalis from various clinical samples.

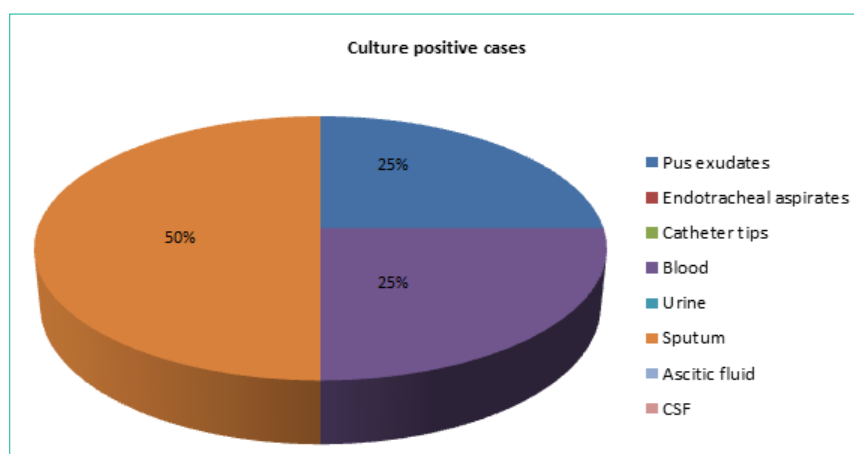

Figure 5: Distribution of isolates of Stenotrophomonas maltophilia from various clinical samples.

positive cases was 56 out of 200 cases reported for hospital association infections and 4 out of 50 cases reported for community association infections. The highest rate of non-fermenters (93.33\%) isolated from the patients admitted into the hospital. The lowest prevalence was seen in patients attended to OPD (6.67\%). Among 60 different non-fermenters were isolated, the predominant non-fermenter was Pseudomonas aeruginosa 40 (66.67\%), followed by Acinetobacter baumannii 10 (16.67\%), Alcaligenes faecalis $6(10 \%)$ and Stenotrophomonas maltophilia 4 (6.66\%). Among 40 Pseudomonas aeruginosa isolates, the highest rate of Pseudomonas aeruginosa isolated from pus exudates $12(30 \%)$ and lowest rate isolated from ascitic fluid and other body fluids 2 (5\%) (Figure 2).

The isolated Pseudomonas aeruginosa differentiated from other species like P. fluorescens, P. putida, P. stutzeri and P. luteola by

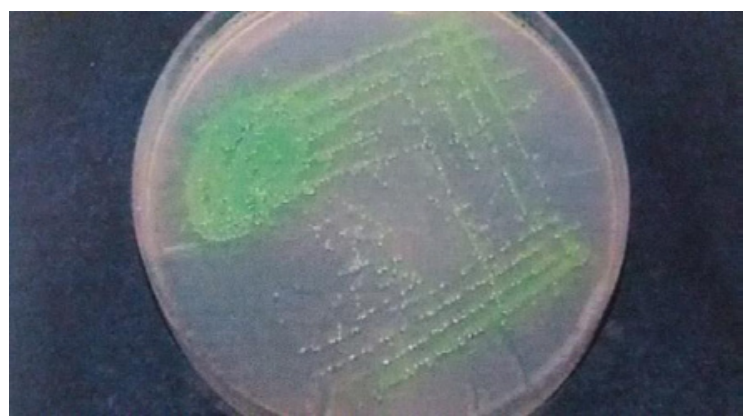

Figure 6: Cetrimide agar plate showing yellow fluorescence of the colonies of Pseudomonas aeruginosa.

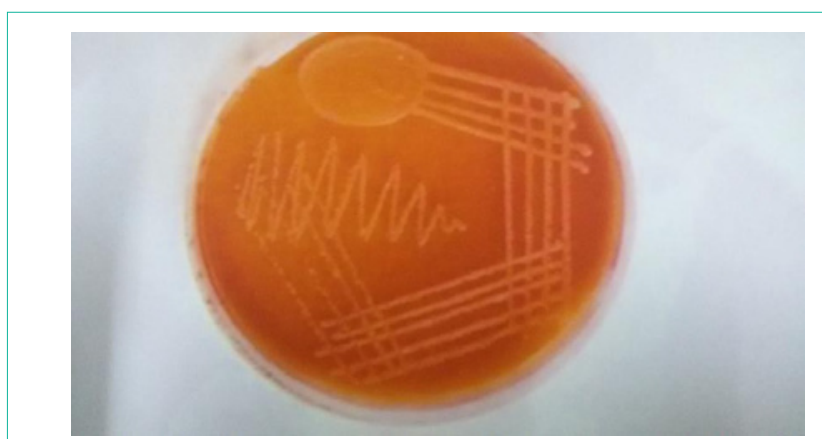

Figure 7: MacConkey agar plate showing growth of Acinetobacter baumannii.

some characteristic features like Oxidase positive, fruity grape like odour, production of large colonies with metallic sheen, mucoid, pigmented (pyocyanin) colonies showing $\beta$-hemolysis. All the isolated non-fermenters were differentiated by using various cultural and biochemical tests (Table 2, Figure 3-7).

The overall antibiotic sensitivity pattern of Non-fermenters isolated from various clinical samples revealed that imipenem was most effective (Figure 8), followed by ceftazidime, piperacillin/ tazobactum, ceftriaxone, cefoperazone, ciprofloxacin, amikacin and gentamicin. Imipenem resistant isolates showed sensitive to polymyxin-B (300U) (Table 3). Among carbapenem resistant isolates of non-fermenters, $100 \%$ showed Metallo-betalactamase production (Figure 9).

\section{Discussion}

The present study was undertaken to evaluate the role of Non-

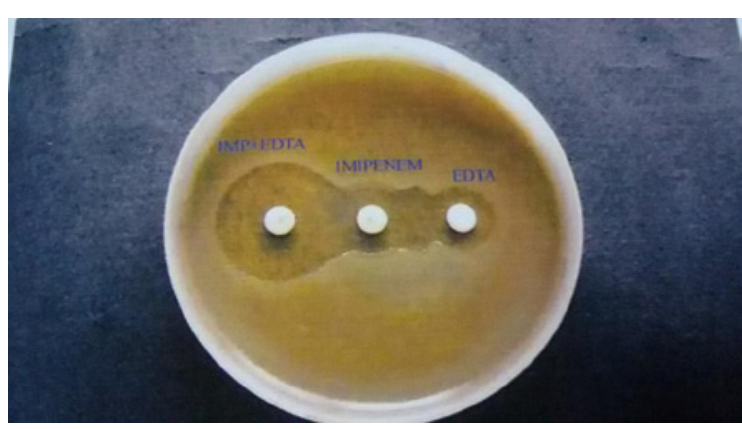

Figure 8: Combined disc test showing MBL production by Alcaligenes faecalis. 
Table 3: Antibiotic susceptibility pattern of the isolates.

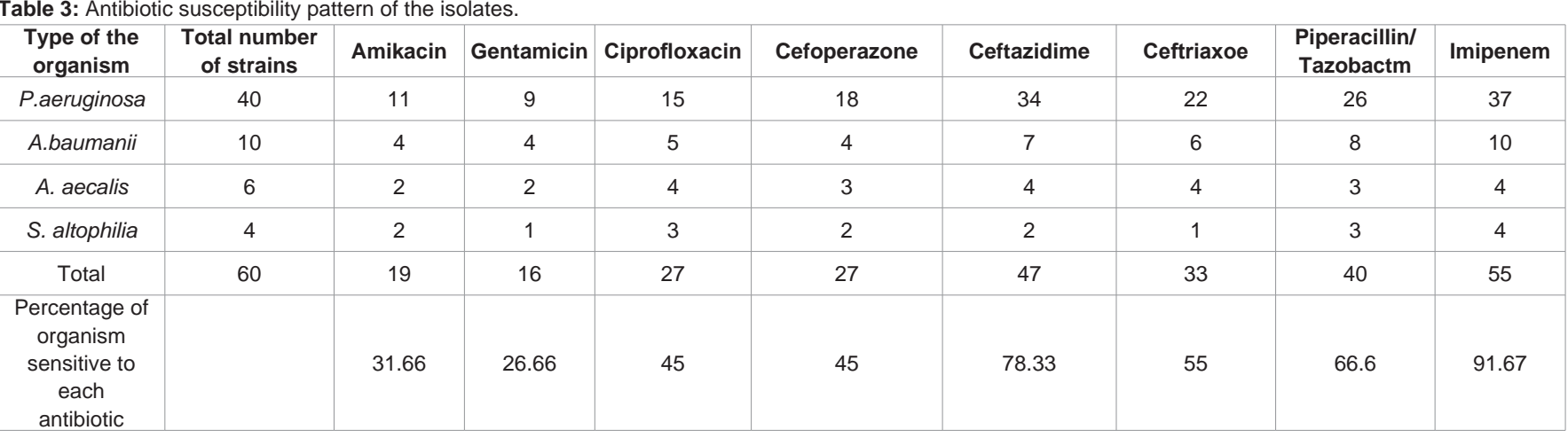

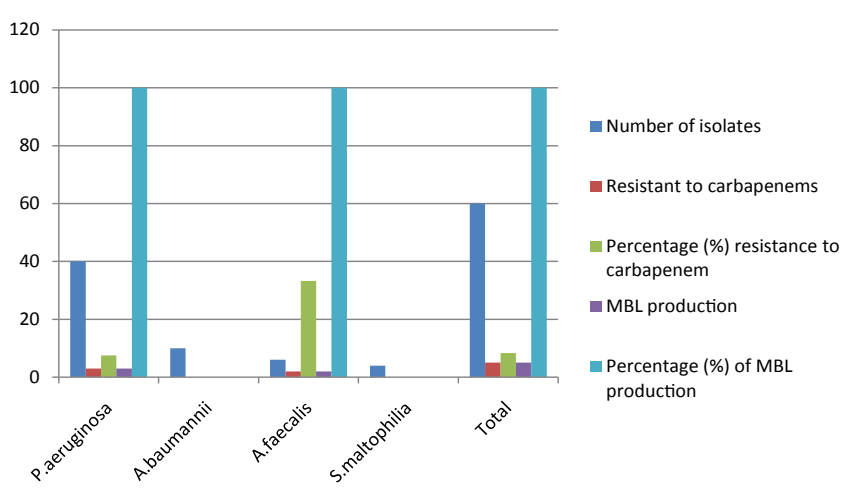

Figure 9: Susceptibility of isolates to carbapenem in relation to MBL production.

fermentative gram negative bacilli in causing various infections especially in hospitalized patients and to know the prevalence of Metallo-betalactamase producing Non-fermenters. Out of 250 different samples, rate of recovery of non-fermenters is $24 \%$ which is comparable with the study of Pathmanathan et al. (24.8\%) [9] and Shail Preet Sidhu et al. (28\%) [10]. Mahajan et al. (80.78\%) [11], Dey and Bairy (74.9\%) [12] reported a higher rate of isolates, whereas Lahiri et al. reported a lower rate of isolates (12.9\%) [13]. In the current study, males were found to be more commonly affected (64\%) with different infections caused by non-fermenters than females (36\%) which is comparable to the findings of Varaiya et al. who observed that $66.66 \%$ were males and $33.3 \%$ were females [14]. Nan-Yao Lee et al. reported $67.4 \%$ were males and $32.6 \%$ were females [15]. Prashanth et al. also found that males were more commonly affected (58\%) than females (42\%) [16]. Different types of infections seems to be more common in males than females, and this male preponderance may be due to that males are more involved with outdoor activities and therefore chances of exposure to infections are more likely. An observation of the source of infection in the present study was indicated that the rate of isolation of Non-fermenters was higher among the hospital acquired infections (93.33\%) than in community acquired infections (6.67\%). Our observation is comparable with the study of Lahiri et al. [13] who showed higher incidence of non-fermenters (82.9\%) in hospital acquired infections than in community acquired infections (17.1\%). Among the isolated non-fermenters Pseudomonas aeruginosa was the commonest isolate $(66.67 \%)$ which coincides with the study of Arjun srinivasan et al. (66.75\%) [17] and Mahajan et al. (54.54\%) [11]. Pathmanathan et al. [9] reported a lower rate of isolates (7.2\%) and also by Neelam Kaistha et al. (11.5\%) [18]. Acinetobacter baumannii was the second most common non-fermenter (16.67\%), in our study. This observation is similar to that of Pathmanathan et al. (17.6\%) [9]. The higher incidence of the isolate was reported by Neelam Kaistha et al. (30.76\%) [18] and also by Dey and Bairy (47.9\%) [12]. 10\% of Alcaligenes faecalis were obtained in the present study where as Shali Preet Sidhu et al. [10] reported only $2 \%$ and Seeta et al. reported only $1.45 \%$. 6.66\% Stenotrophomonas maltophilia was reported in the present work but Shali Preet Sidhu et al. [10] reported only 1\%. Regarding the antibiotic susceptibility pattern, the highest sensitivity rate was observed with Imipenem (91.67\%) and it coincides with the study of Duggal et al. (95.74\%) [19] and Agarwal et al. (90.5\%) [7].The least sensitivity was observed to gentamicin $(26.66 \%)$ in our study. In the present investigation, $7.5 \%$ of the Pseudomonas aeruginosa proved to be resistance to the carbapenems and all the carbapenem resistant strains were MBL producers (100\%). Shali Preet Sidhu et al. [10] reported that $6.97 \%$ of the Pseudomonas aeruginosa showed resistance to the carbapenems, and it is coinciding with the present study. Irfan et al. [4] reported (100\%) of the carbapenem resistant isolates of Pseudomonas aeruginosa were MBL producers, which are also coinciding with the study. All the pathogenic cultures which were isolated in the current investigation showed $100 \%$ sensitivity to Polymyxin-B. Antibiotic options are rather limited in multipleresistant NFGNB infections; old but well known Polymyxins, which have become available again, are an option in the treatment of resistant strains and now-a-days widely used in clinical practices for NFGNB infections. Many unusual organisms were involved in the hospital association infections had numerous predisposing factors such as immunosuppression in the case of cancers, diabetes, chronic diseases, prolonged hospital stay, different surgical conditions and continuous antibiotics usage were also contribute to morbidity and mortality. Though they were not frequently isolated like other bacteria, they hoist significant therapy issues throughout intrinsic resistance to multiple antibiotics classes, and increased acquired rate of resistance factors that determine their rapid propagation in the hospital. Education of health care staff recommending medication, hospital infection control, antibiotic management, execution of an active surveillance system, limit of non-human use of antimicrobials and distribution of data about resistant strains at national and international level are suggested to control resistant bacteria. The world wide resistance pattern of nosocomial pathogens illustrates wide variation from country to country and within the same country over a period of time. Appropriate identification and detection of antibiotic susceptibility pattern is of huge value because of high 
intrinsic resistance of different NFGNB to various antimicrobial agents. Therefore, different international authorities put emphasis on every hospital should have antibiotic policy of its own [20].

\section{Conclusion}

Non-fermenters are emerging as nosocomial pathogens and they show Multidrug resistance, so the excess use of carbapenems and other broad spectrum antibiotics should be avoided and also early detection and prompt infection control measures are important to prevent further spread of MBLs to other Gram negative bacilli.

\section{References}

1. Washington W, Stephen A, Janda W, Koneman E, Procop G, Schrekenberger $P$, et al. Text book of diagnostic microbiology. $6^{\text {th }}$ Edition. Philadelphia Wolters kluwer Health. 2006.

2. Connie Mahon R, Donald Lehman C, Manuselis G. Text book of diagnostic microbiology. $2^{\text {nd }}$ Edition. Elsevier: Church hill Livingstone. 2000.

3. Seetha CS, Bairy I, Shivananda PG. Bacteraemia in high risk patients. Indian J Med Microbiol. 2002; 56: 391-396.

4. Irfan S, Zafar A, Guhar T, Ahsan Hosan R. MBL producing clinical isolates of Acinetobacter species and Pseudomonas aeruginosa from ICU patients of a Tertiary care hospital. Ind J Med Micribiol. 2008; 26: 243-245.

5. Collee JG, Fraser AG, Marmion BP, Simmons A. Mackie and McCartney Practical Medical Microbiology. 14th Edition. Elsevier: Church hill Livingstone. 2006.

6. Mechawal SK, Taneja N, Sharma SK, Meera Sharma. Complicated nosocomial UTI caused by non-fermenters. Ind J Urology. 2002; 18: 123-128.

7. Agarwal VA, Dongre SA, Powar RM. Antimicrobial resistance profile of metallo beta lactamase Pseudomonas aeruginosa producing metallo beta lactamases. Indian J Med Res. 2006; 124: 588-590.

8. Behera B, Mathur P, Das A, Kapil A, Sharma V. An evaluation of 4 different pherotypic techniques for detection of $\mathrm{MBL}$ producing Pseudomonas aeruginosa. Indian J Med Microbiol. 2008; 26: 233-237.

9. Pathmanathan SG, Samat NA, Ramelah M. Antimicrobial susceptibility of clinical isolates of Pseudomonas aeruginosa from a Malaysia hospital. Malaysia J Med Sci. 2009; 16: 27-32.
10. Shail Preet Sidhu, Usha Arora, Pushpa devi. Non-fermenting gram negative bacilli- An important cause of bacteremia in immunocompromised patients. JK Science. 2010; 12: 168-171.

11. Mahajan R, Neeraj, Sarika, Mahajan B. Isolation and identification of nonfermenting Gram negative bacilli in a Tertiary care hospital. Sch J Appl Med Sci. 2016; 4: 872-876.

12. Dey A and Indira Bairy. Incidence of multi-drug resistant organisms causing ventilator associated pneumonia in a Tertiary care hospital. A 9 months prospective study. Ann Thorac Med. 2007; 2: 52-57.

13. Lahiri KK, Mani C. Acinetobacter species as nosocomial pathogen: Clinical significance and Antibiogram sensitivity. Med J Armed Forces India. 2004; 60: 7-10.

14. Variaya A, Kulkarni M, Bhalekar, Dorga J. Incidence of carbapenem resistant Pseudomonas aeruginosa in Diabetes and Cancer patients. Ind $\mathrm{J}$ Med Microbiol. 2008; 26: 238-240.

15. Nan-Yao Lee, Nai-Ying Ko RN, Chang CM. Clinical and Economic impact of Multidrug resistance in nosocomial Acinetobacter baumannii bacteremia. Infection control and hospital epidemiology. 2007; 28: 713-719.

16. Prashanth K, Badrinath S. Nosocomial infections due to Acinetobacte species: Clinical findings, risk and prognostic factors. Ind J Med Microbiol. 2006; 24: 39-44.

17. Srinivasan A, Linda L, Karen M, Hartsell TL, Jones HD, Diette GB, et al An outbreak of Pseudomonas aeruginosa infections associated with flexible bronchoscopes. Ind J Med Microbiol. 2003; 348: 221-227.

18. Kaistha N, Garg R, Mehta M, Chander J, Singla N. Neonatal septicemia isolates and resistance patterns in a Tertiary care hospital of north India. J Infect Dev Ctries. 2010; 4: 055-057.

19. Duggal S, Khatri PK, Parihar RS. Antibiogram of various bacterial isolates from pus samples in a Tertiary care centre in Rajasthan. Int J Sci Res. 2013. 4: $1580-1584$.

20. Benachinmardi KK, Padmavathy M,Malini J, Naveneeth BV. Prevalence of non-fermenting Gram negative bacilli and their in vitro susceptibility pattern at a tertiary care teaching hospital. J Scientific society. 2014; 41: 162-166.
J Bacteriol Mycol - Volume 4 Issue 1 - 2017

ISSN : 2471-0172 | www.austinpublishing group.com

Vijayalakshmi et al. (C) All rights are reserved
Citation: Usha Rani P and Vijayalakshmi P. Epidemiological Pattern of Non-Fermenting Metallo Beta Lactamase Producing Bacterial Pathogens Isolated From Clinical Specimens in a Tertiary Care Hospital in Kakinada. J Bacteriol Mycol. 2017; 4(1): 1043 